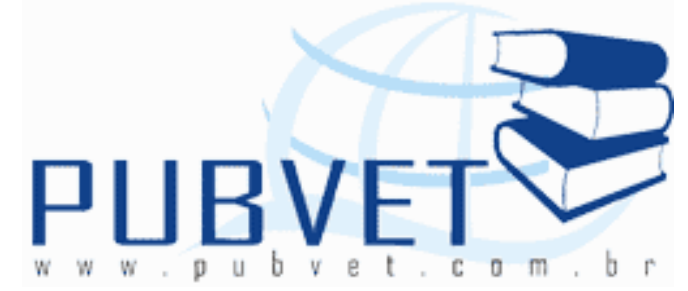

PUBVET, Publicações em Medicina Veterinária e Zootecnia.

\title{
A síndrome da cauda equina em cães: revisão de literatura
}

\author{
FERREIRA, Luiz F. L. e SANTOS, Fabíola F.
}

\section{Resumo}

O tema do presente artigo se refere à Síndrome da cauda eqüina em cães com ênfase no tratamento da doença. A cauda eqüina é uma coleção de raízes de nervos que descem o canal vertebral até o final da medula espinhal. Esta síndrome acomete principalmente cães de grande porte. Existem vários graus de envolvimento dos membros pélvicos, bexiga, esfíncter anal e cauda, com sinais clínicos variando desde flacidez até a paralisia dos membros pélvicos. 0 tratamento recomendado pela maioria dos autores é cirúrgico, pela técnica de laminectomia, porém não se descarta a possibilidade de utilizar o tratamento conservativo. Tratamentos alternativos, como a fisioterapia, acupuntura e quiropraxia, são utilizados cada vez mais pela Medicina Veterinária, associados e como suporte aos tratamentos convencionais. O prognóstico é bom para os animais que não apresentam disfunção urinária e fecal.

Palavras-chave: cães, cauda eqüina, laminectomia.

\section{The cauda equine syndrome in dogs: literature review}

\section{Abstract}

The theme of this article is the equine tail syndrome in dogs with emphasis on treatment of the disease. The equine tail is a set of nerve roots that descends 
through the spinal meatus to the end of the spinal cord. This syndrome strikes mainly in large dogs. There are many degrees of involvement of hindlimbs, bladder, anal sphincter and tail, with clinical signs that vary from flabbiness until loss of the power of voluntary motion of the hindlimbs. The most authors recommend surgery by using Laminectomy, however, the conservative treatment is not ruled out. Veterinary medicine uses alternative treatment as (physioterapy), acupuncture and Chiropraxy associated to conventional treatments.

Keywords: dogs, equine tail, laminectomy.

\section{INTRODUÇÃO}

A síndrome da cauda eqüina é a enfermidade neurológica provocada por estenose congênita ou adquirida do canal vertebral lombossacro (SCHULMAN; LIPPINCOTT, 1990). Esta síndrome reflete vários graus de envolvimento dos membros pélvicos, bexiga, esfíncter anal e cauda, com sintomas clínicos que variam desde flacidez até paralisia dos membros pélvicos (BOJRAB, 1996). O presente trabalho trata dos distúrbios detectados com maior freqüência na Medicina Veterinária.

Os sinais clínicos da síndrome da cauda eqüina diferem-se daqueles observados em outras localizações da medula espinal, em virtude da estrutura anatômica particular da região, a condição é vista em cães de várias idades e rara em gatos (WHEELER; SHARP, 1999).

Graus diferentes de lesões podem estar presentes, resultando em diversos métodos de tratamento, baseados principalmente no conhecimento clínico do médico veterinário e não em dados de literatura (SEIM III, 2002).

\section{ESTRUTURA DA CAUDA EQUINA}

A cauda eqüina é um feixe de nervos contidos no interior do canal espinhal da coluna vertebral lombar inferior e sacral (PRATA, 1998). Ela contém grande número de raízes nervosas em uma pequena área localizada 
em L6 até S3 e, por isso, uma lesão na região pode envolver vários nervos (BOJRAB, 1996).

Nos cães, os últimos segmentos da medula espinhal lombar, L5, L6, L7, encontram-se no interior da quarta vértebra lombar. Os segmentos sacrais $\mathrm{S} 1$, S2, S3 localizam-se dentro do corpo da quinta vértebra lombar e os segmentos coccígeos dentro da sexta vértebra lombar (NELSON \& COUTO, 2006). As raízes dos nervos L6 e L7 e S1 formam o nervo isquiático. Os nervos S2 e S3 contribuem para a formação do nervo podendo, que inerva o períneo e o esfíncter anal externo, além do nervo pélvico, que controla a continência urinária e fecal (PRATA, 1998).

\section{SÍNDROME DA CAUDA EQUINA}

A síndrome da cauda eqüina é definida como a moléstia neurológica provocada por estenose congênita ou adquirida do canal vertebral lombossacro (SCHULMAN; LIPPINCOTT, 1990). São sinônimos de síndrome da cauda eqüina: estenose lombossacra, compressão da cauda eqüina, espondilose lombossacra, malformação ou má-articulação lombossacra e instabilidade lombossacra (BOJRAB, 1996). Via de regra ocorre em cães de grande porte, especialmente da raça Pastor Alemão, Border Collie e Labrador Retriever (BRASIL, 2006) e acomete mais machos do que fêmeas, entre os 2 e 13 anos (CHRISMAN, 1985).

\section{CAUSAS DE SÍNDROME DA CAUDA EQUINA}

A síndrome da cauda equina pode ser congênita ou adquirida. A causa congênita é rara, ocorrendo geralmente em cães com acondroplasia. Já, a etiologia adquirida sucede geralmente a extrusão de disco, estenose do canal devido à espondilose crônica, fraturas e luxações, que determinam a compressão da região (BOJRAB,1996). Pode ocorrer secundariamente a tumor, osteocondrose vertebral ou sacral, malformação óssea congênita ou proliferação progressiva de tecidos da região lombossacra (NELSON \& COUTO, 
2006). As causas mais comuns de síndrome da cauda equina estão listadas a seguir.

\section{1 Doença do disco intervertebral L6-L7}

A doença do disco intervertebral (DDIV) é um enrijecimento prematuro do centro do disco associado a uma debilidade da parte externa ou ânulo. A degeneração do disco intervertebral ocorre com a idade e pode preceder a herniação discal Hansen (1952) apud Wheeler e Sharp (2005). As lesões discais podem ser definidas classicamente como degeneração condróide e fibróide:

\subsubsection{Metaplasia condróide (TIPO I)}

A Metaplasia Condróide ocorre geralmente em raças condrodistróficas, como Shih Tzu, Beagle, Basset Hound, Teckel, entre outras, nos primeiros dois anos de vida. Como conseqüência da fragilidade do ânulo fibroso, associada à mineralização de seu núcleo, ocorre a extrusão do material discal para o canal medular e a herniação do tipo Hansen I ou extrusão discal (GARIBALDI, 2003).

\subsubsection{Metaplasia Fibróide (Tipo II)}

Ocorre normalmente em raças não condrodistróficas após a meia idade, como animais de grande porte. $O$ núcleo pulposo do disco intervertebral destes animais também sofre um processo de desidratação, porém este é invadido por tecido fibrocartilaginoso e não cartilagem hialina. A predisposição genética, a conformação, a atividade física e as malformações vertebrais são fatores propostos como causa de aumento no estresse mecânico no disco intervertebral na junção lombossacra, provendo doença do disco intervertebral.

\subsection{Traumas}

O trauma pode resultar em fratura ou luxação de L7, L7-S1, sacro ou junção sacrococcígea. Entretanto, fraturas do corpo vertebral L7 são 
relativamente comuns, normalmente como o resultado de um acidente automobilístico (PRATA, 1998).

\subsection{Discoespondilite}

Também denominada de osteomielite intradiscal é uma doença que envolve um ou mais discos e suas vértebras adjacentes, que pode estar associado à infecção do trato urinário, endocardite, dermatite, orquite ou bacteremias, podendo ser resultado de infecção por fungo ou bactéria, devido à migração de corpo estranho, anestesia peridural ou até mesmo via hematógena, sendo o Staphylococcus aureus o microrganismo mais freqüente nestes casos (McKEE et al. 1990).

\subsection{Hemangiosarcoma de meninges}

É uma neoplasia maligna de origem das células endoteliais, caracterizada por metástases precoces e agressivas e que pode ter origem em qualquer tecido vascularizado. Pode acometer a meninge levando a compressão da medul. (PINTO, 2007).

\subsection{Espondilose}

Ela ocorre devido à formação de pontes ou esporões ósseos laterais ou ventrais ao corpo vertebral, levando à compressão medular, sem causa inflamatória ou infecciosa, podendo estar associada com hérnia ventral, ou instabilidade vertebral, sendo um achado comum em cães de grande porte (PRATA, 1998).

\subsection{Estenose Degenerativa Lombosacral}

É a forma mais comum de patologia lombosacra e acomete principalmente cães adultos de grande porte. Resulta em protrusão discal do tipo II com protuberância dorsal do anel fibroso, hipertrofia/hiperplasia do ligamento arqueado, formação osteofítica, espessamento dos arcos 
intervertebrais ou facetas articulares, e raramente subluxação/instabilidade da junção lombosacra (MORIKAWA, 2011).

\subsection{Estenose Lombosacra Idiopática}

Diferente da Estenose Lombosacra Degenerativa é uma doença definida de cães de pequeno e médio porte resultante de pedículos curtos e espassados, lâminas e facetas articulares escleróticas e espassadas, espassamento e envolvimento do ligamento longitudinal e facetas articulares invadindo dorsalmente o canal vertebral. Acredita-se que estas alterações resultam de uma falha no desenvolvimento do arco neural (MORIKAWA, 2011).

\subsection{Neoplasias}

Independente do tecido de origem, neoplasias da coluna vertebral resultam em invasão do tecido nervoso no canal vertebral, sendo que as massas extradurais representam aproximadamente $50 \%$ dos tumores espinhais. Embora os tumores de origem óssea sejam os mais comuns, metástases de adenocarcinomas prostáticos e mamários também podem ocorrer (MORIKAWA, 2011).

\section{MÉTODOS DIAGNÓSTICOS}

\subsection{Sinais Clínicos}

Os cães acometidos exibem lentidão para se levantar de uma posição inclinada e ainda relutância em correr, sentar, saltar, subir escadas ou abanar a cauda (PALMER; CHAMBERS, 1991; KONNO et al. 1996). A claudicação e a fraqueza dos membros pélvicos agravam-se com os exercícios, pois os vasos sanguíneos que acompanham as raízes dos nervos espinhais dentro do forame intervertebral já comprimido dilatam-se e comprimem ainda mais as raízes nervosas (PALMER; CHAMBERS, 1991).

Pode ocorrer hiperestesia ou parestesia do períneo, com dermatite úmida auto-infligida do períneo e da base da cauda. As incontinências urinárias e 
fecais são raras (NELSON \& COUTO, 2006; RAMIREZ \& THRALL, 1998). A bexiga é freqüentemente paralisada, que resulta em retenção de urina e passivas transbordações, ou seja, incontinência (JEFFERY, 1995).

Com freqüência, os achados clínicos representam a base para se obter o diagnóstico em cães acometidos, devido à dificuldade de interpretação de muitos testes diagnósticos de rotina. Para Nelson \& Couto (2006) o achado mais consistente do exame físico é a dor eliciada por palpação profunda da região dorsal do sacro ou por dorsoflexão da cauda ou hiperextensão da região lombosacra.

Para Seim III (1997) os reflexos miotáticos espinhais avaliam a integridade dos componentes sensoriais e motores do arco reflexo e a influência dos trajetos motores descendentes no reflexo, podendo-se obter três níveis de resposta: a) ausência de reflexos; b) reflexos normais; e c) reflexos exagerados.

Como regra geral deve-se realizar o teste sensorial por último, que deve ser pela aplicação de estímulos dolorosos em cada membro e na cauda. Devem-se usar estímulos dolorosos progressivamente mais fortes, como pinça hemostática Kocker, para avaliar a percepção dolorosa profunda. Como regra geral, a perda de função após uma lesão no cordão espinhal se desenvolve como se segue: (1) perda da propriocepção, (2) perda de função motora voluntária, (3) perda de sensação dolorosa superficial e (4) perda de sensação dolorosa profunda (SEIM III,1997).

\subsection{Métodos Diagnósticos por Imagem}

Dentre os métodos de diagnóstico por imagem, não há um que seja capaz de consistentemente diagnosticar a síndrome da cauda equina. Em consequência disso, múltiplos métodos diagnósticos têm sido utilizados para avaliar essa patologia (RAMIREZ \& THRALL, 1998). 


\subsubsection{Exame radiográfico}

As radiografias espinhais são úteis para descartar causa de compressão de cauda equina como discoespondilite, neoplasia vertebral lítica, fratura/luxação, e identificar os fatores predisponentes para a estenose degenerativa tais como, osteocondrose sacral, malformações vertebrais (SEIM III, 1997).

\subsubsection{Mielografia}

A mielografia é em geral necessária para um diagnóstico definitivo de doença de discos intervertebrais, quando se antecipa uma cirurgia descompressiva. A indicação de mielografia ocorre nos seguintes casos: Quando não se identifica lesão visível em radiografias de pesquisa; quando se observam lesões múltiplas compatíveis com o exame neurológico; ou quando se visualiza lesão incompatível com o exame neurológico (SEIM III, 1997).

\subsubsection{Eletromiografia}

Quando disponível, segundo Nelson \& Couto (2006), a eletromiografia (EMG) da musculatura dos membros pélvicos, da região paraespinhal e da cauda pode sugerir desenervação dos músculos inervados pela cauda equina, confirmando uma suspeita clínica de compressão desse segmento medular. A EMG também pode ser usada para detectar polimiosite e polineuropatia em cães cujo diagnóstico é questionável.

\subsubsection{Ressonância Magnética}

A imagem por ressonância magnética (IRM) é mais útil no diagnostico de estenoses lombossacrais e tumores intramedulares. As desvantagens da IRM são o custo, a inacessibilidade e a má resolução dos ossos tensos ou corticais (SEIM III,1997). A alta resolução da RM permite obter imagens claras da cauda equina. A sua utilização dispensa a necessidade de contrastes e é capaz de evidenciar a cauda equina e tecidos moles na altura de L7- S1. Também é 
possível evidenciar a gordura epidural, não visível na radiografia e mielografia (TAGA, 1998). O uso da RM para caracterizar os componentes anatômicos da compressão é especialmente importante ao se ponderar o tratamento cirúrgico (NELSON \& COUTO, 2006).

\subsubsection{Epidurografia}

A Epidurografia é realizada mediante a injeção do meio de contraste no espaço epidural (BRAWNER, 1995). É uma das modalidades de diagnóstico por imagem comumente utilizada para a avaliação de doença lombossacra em cães. Isto porque, na maioria dos cães, o saco dural termina cranial a junção lombo sacral, tornando a mielografia de valor limitado na avaliação desta região (TAYLOR, 1998).

\section{DIAGNÓSTICO DIFERENCIAL}

Os principais diagnósticos diferenciais são: afecções neurológicas tais como, discoespondilite, neoplasia, anomalia congênita, mielopatia degenerativa, neurite da cauda eqüina (BRASIL, 2006); afecções ortopédicas tais como, artrite coxo-femoral, displasia coxo-femoral, ruptura do ligamento cruzado, contratura do grácil, e prostopatias (WHEELER, 1999).

\section{TRATAMENTO}

Diversos tratamentos têm sido descritos, desde acupuntura, repouso, uso de antiinflamatórios e procedimentos cirúrgicos (CHIERICHETTI e ALVARENGA, 1999). O tratamento mais adequado depende do estado neurológico do animal, do histórico médico e da evolução dos sinais clínicos, por isso cada caso deve ser avaliado individualmente (DE LAHUNTA, 1983; LECOUTEUR e CHILD, 1992; SIMPSON, 1992).

\subsection{Tratamento Conservativo}

Jeffery (1995) cita que existem tratamentos conservativos, à base de costicosteóides, antiinflamatórios não esteroidais e acupuntura, mas que esses 
não apresentam de uma forma geral resultados satisfatórios. As abordagens primárias incluem o uso de antiinflamatóriosnão esteroidais (AINES), os quais atuam apenas na dor nociceptiva. Nas dores neuropáticas, uma abordagem terapêutica pode ser utilizada, tais como o uso de antidepressivos como amitriptilina, anticonvulsivantes como a gabapentina, carbamazepina e pregabalina e opióides. Dessa forma, a combinação desses componentes analgésicos com AINES pode ser útil no caso de dor crônica não responsiva aos tratamentos apenas com fármacos de ação antinociceptiva (BRIDGES et al. 2001; HELLYER et al. 2007).

\subsection{Tratamento Cirúrgico}

\subsubsection{Técnica cirúrgica}

A cirurgia deve ser indicada após exame neurológico seriado e de acordo com o estado neurológico do paciente e pela resposta à terapia médica conservativa (SEIM III,2002).Apesar de existirem várias técnicas para descompressão da cauda eqüina como a técnica de fenestração, discectomia, fixação ou fusão, a laminectomia é a técnica mais indicada, onde o animal deve ser posicionado dorso-ventralmente (PRATA, 1998).

\subsubsection{Fenestração}

A fenestração é realizada como um meio profilático combinado ao procedimento descompressivo ou seguida de um bem sucedido tratamento clínico. Terapeuticamente é realizada em animais apresentando somente dor no dorso ou leve paresia associada à protrusão discal (SHORES, 1985). Não é indicada para pacientes com material discal que tenha herniado para o interior do canal espinhal ou do forame intervertebral; no entanto, pode beneficiar paciente com dor discogênica (SEIM III, 1997). Ela é eficaz quando discos de degeneração protruem e causam radículas e fibras nervosas, que respondem pela maioria dos problemas discais cervicais (BOJRAB e CONSTANTINESCU, 1996). 
Segundo Brisson et al. (2004), fenestração do disco herniado simultaneamente à descompressão é recomendada para prevenir extrusão contínua do material do disco degenerado no período pós-operatório podendo resultar em decorrência precoce dos sinais clínicos. SEIM III (1997) não recomenda a fenestração discal após procedimento de fenda ventral.

\subsubsection{Laminectomia}

Segundo SEIM III (1997), a laminectomia é indicada quando as lesões se localizam no canal espinhal dorsal e lateral. A laminectomia dorsal modificada é indicada para a exposição de massas compressivas nas facetas ventrolateral e dorsal do canal espinhal (fragmentos discais, fragmentos de fratura, ressecção de neoplasias vertebrais, de cordão espinhal, de raízes nervosas e exposição de fraturas/luxações). Usando essa técnica, deve-se realizar laminectomia de não mais que duas vértebras consecutivas.

A laminectomia é recomendada quando a lesão por compressão discal é caudal ao segmento espinhal L3. (AMSELLEM, 2003). A laminectomia dorsal deve ser usada mais comumente para descomprimir a estenose lombossacral (ou seja, síndrome da cauda eqüina), expor e remover material discal herniado, fragmentos de fratura, neoplasias ou abcessos paraespinhais em L6L7 ou L7- S1 (SEIM III, 1997).

Antes do fechamento, avalia-se a espinha quanto à estabilidade. Teoricamente, a descompressão deve se limitar à região que provoca a disfunção neurológica específica, preservando-se assim tantos tecidos moles e estruturas estabilizadoras ósseas quanto possível. As facetectomias, na junção lombosacral podem criar instabilidades que podem levar a uma futura recorrência da doença. Uma instabilidade grosseira é raramente uma característica nos pacientes com estenose do canal espinhal (BOJRAB, 1996). Deve-se lavar o local com solução salina morna, e logo após deve ser coletada gordura subcutânea livre para funcionar como enxerto, a gordura é posicionada em cima do local da laminectomia, os músculos epaxial internos 
são suturados com monofilamento não absorvíveis, e os tecidos subcutâneos e pele são suturados de maneira rotineira (JEFFERY, 1995).

\subsubsection{Técnica de Fixação-Fusão L7-S1}

A técnica estira o anel redundante do disco L7-S1, com o objetivo de aliviar a compressão sobre a cauda eqüina, fixando as vértebras na posição distendida, e permite que a consolidação óssea promova a fusão das vértebras nesta posição. (SLOCUM, 1986)

O cão é posicionado em decúbito ventral, com os membros estendidos cranialmente. O cirurgião faz uma incisão na linha média dorsal, desde L6 até a primeira vértebra coccígea. A fáscia superficial e a fáscia sacral profunda da subjacente são igualmente submetidas a incisão, paralelamente à incisão cutânea. A musculatura epaxial é elevada dos processos espinhosos dorsais e lâminas de L7 até S3, sendo em seguida rebatida lateralmente. Em cães sofrendo compressão da cauda eqüina, ocorre o colapso do espaço interarciforme entre L7 e S1. O espaço interarciforme é expandido até que as cartilagens articulares das facetas fiquem alinhadas na posição normal. $O$ ligamento amarelo é incisionado e rebatido, para que ocorra a exposição do tecido adiposo e nervos sacrais no canal espinal. Os nervos sacrais são mobilizados, para a observação da parte dorsal do anel fibroso. (SLOCUM, 1986)

Um orifício é perfurado com broca de $2,5 \mathrm{~mm}(2,0 \mathrm{~mm})$ através da faceta articular caudal direita, até o sacro. O orifício é medido e rosqueado; a seguir, é aplicado um parafuso cortical de $3,5 \mathrm{~mm}(2,7 \mathrm{~mm})$. Um segundo orifício é perfurado através da faceta articular caudal até o sacro. É aplicado um parafuso cortical com o comprimento apropriado. (SLOCUM, 1986)

O espaço interarciforme é revestido com tecido adiposo e esponja de gelatina absorvível. É coletado de ambas as asas do ílio, enxerto ósseo, aproveitando a incisão cutânea original. Assim, a fáscia do sacro é lateralmente afastada, e os músculos sacrocaudais são afastados medialmente. O músculo iliocostal e o longo lombar são elevados da parte medial da asa do 
ílio. Usando osteótomo, é criada janela na superfície medial da asa do ilíaco. Com uma cureta obtem-se osso corticotrabecular do lado iterno de ambas as asas do ílio. O córtex lateral e a margem do ílio são deixados intactos. O enxerto ósseo é aplicado sobre as lâminas, desde a sétima vértebra lombar até o sacro. A oclusão se processa por técnica de rotina. A musculatura epaxial é suturada de modo que o enxerto ósseo seja mantido no lugar. A fáscia sacral profunda é suturada, e também as camadas subcutâneas e a pele. (SLOCUM, 1986)

\subsection{Tratamentos Alternativos}

\subsubsection{Acupuntura e eletroestimulação}

A acupuntura é indicada para problemas funcionais como paralisia, processos inflamatórios não infecciosos, possui efeito sobre os sistemas autônomo, nervoso, analgésico e antiinflamatório. (MALMEGRIN, 2007). É a técnica de estímulo de pontos específicos no organismo, ativados de diversas formas, como a inserção de agulhas, calor, implantes de ouro, laser, injeção de vitaminas ou fármacos, entre outras (SCHOEN, 2006).

Segundo Brunner (1976) a eletroestimulação é um método necessário para tratar doenças neuromusculares. A estimulação elétrica foi utilizada como tratamento auxiliar na recuperação dos movimentos em pacientes humanos com lesão de medula. (WONG et al., 2003). A associação da eletroestimulação à acupuntura manual pode ser baseada na atrofia muscular do paciente, na gravidade dos sinais neurológicos e no tempo de paresia ou ataxia (JOAQUIM et al., 2003).

\subsubsection{Associação do tratamento conservativo com acupuntura}

O pré-tratamento com antiinflamatórios não esteróides, vitaminas seguido de acupuntura foi significantemente melhor do que a acupuntura de forma isolada (STILL, 1988a; STILL, 1988b; STILL, 1989). Injeções locais nos pontos de acupuntura também podem ser utilizadas. Nestes casos pode-se injetar lidocaína 0,25\%, procaína, cafeína, vitaminas B1, B2, B6, B12 e dimetil 
sulfóxido (DMSO), cloreto de sódio ( $\mathrm{NaCl}$ ), água, hidróxido de sódio $\mathrm{NaOH}$ ), antiinflamatórios não esteróides e esteróides, e soluções homeopáticas (JANSSENS, 2001).

\subsubsection{Fisioterapia Associado ao tratamento conservativo e a acupuntura, e ao pós-operatório}

Em um estudo da Universidade de Konkuk, Korea, os autores relataram 15 casos de cães com discopatia toracolombar tratados de maneira conservativa. Os animais foram tratados com prednisolona, carprofeno e antibióticos, acupuntura (Ji Zhong, Bai Hui, Zhong Shu, Be28, E36 e VB30) e fisioterapia (hidroterapia, termoterapia, massagem, manipulação e natação). Dos 15 animais, 12 recuperaram os déficits neurológicos em 21 dias após o inicio dos tratamentos. Os outros três animais que não melhoraram apresentavam outras enfermidades medulares concomitantes. Todos os animais apresentaram controle urinário normal, após o tratamento e após 21 meses, nenhum dos 12 animais apresentou recorrência. Dessa maneira os autores concluíram que a terapia conservativa associada aos tratamentos alternativos, pode ser efetiva no manejo de animais com paraparesia, paraplegia e disfunções urinárias causadas por doença do disco intervertebral (HYUNJUNG et al., 2003).

A utilização da fisioterapia pós-operatória é de grande valia para a aceleração do processo de recuperação e conseqüentemente qualificando o resultado do procedimento cirúrgico (FREIRE, 2005).

\subsubsection{Quiropraxia}

A quiropraxia é a manipulação da coluna vertebral e das articulações do organismo, objetivando o alinhamento de articulações que estejam mal ajustadas; identifica e procura as causas mecânicas de doenças, onde há pinçamentos e compressões nervosas, causadas por vértebras rotadas ou bloqueadas ou mesmo por tensão muscular. (GLÜTTER, 2007). O médico veterinário quiroprático faz um movimento rápido com as mãos ou com o 
ativador, é o que basta para corrigir uma vértebra fixada ou sub luxada. Após o ajuste da coluna, os membros serão examinados, procurando se há áreas de tensão muscular, áreas mais frias ou mais quentes e checando se a mobilidade das articulações. (ERNST, 2008; ASBACO, 2008; RENALVET,2008).

\section{CONSIDERAÇÕES FINAIS}

O conhecimento das doenças relacionadas à medula espinhal pelo medico veterinário é de extrema importância. Tais patologias possuem origens e modos de ação diferentes, no entanto todas levam a uma diminuição na qualidade de vida deste animal, levando ao sofrimento destes pacientes e seus proprietários, pela lesão neurológica causada podendo até ser indica a eutanásia dependendo do estado do paciente.

Deve ser realizado o diagnóstico diferencial de moléstias não associadas com a junção lombossacra que imitam a síndrome da cauda eqüina. Múltiplas modalidades de imagem estão disponíveis para facilitar o diagnóstico da síndrome da cauda eqüina; porém muitas ainda são inespecíficas e têm baixa sensibilidade dificultando o fechamento do diagnóstico.

Os diferentes tipos de tratamentos para este tipo de lesão necessitam ser mais estudados e aprimorados para aumentar assim, as chances de cura do paciente e alívio da dor, que atualmente, apresenta normalmente, um prognóstico reservado quando diagnosticado com uma lesão mais grave.

\section{REFERÊNCIAS}

1. AMSELLEM, P.M.; TOOMBS, J.P.; LAVERTY, P.H. et al. Loss of deep pain sensation following thoracolumbar intervertebral disk herniation in dogs: pathophysiology. Comp. Cont. Educ. Pract. Vet., v.25, p.256-264, 2003a.

2. ASBACO, Associação Nacional de Quiropraxia Quiropraxia. 2007. Disponível em http://www.quiropraxia.org.br. Acesso em 14 Abril 2011.

3. BOJRAB, M. Joseph et al. Técnicas atuais em cirurgia de pequenos animais. São Paulo: Roca, p.591-597, 1996. xix, 896 p.

4. BRASIL, Fabrício Bernardo de Jesus et al. Síndrome da cauda eqüina, etiopatologia. Revisão de literatura (parte I). Boletim Médico Veterinário, Espírito Santo do Pinhal, v.2, n.2, p.26-33, jan./dez. 2006. 
5. BRIDGES, D.; THOMPSON, S.W.N.; RICE, A.S.C. Mechanisms of neuropathic pain. Br. J. Anaesth., v.87, p.12-26, 2001.

6. BRISSON, B. A.; MOFFATT, S. L.; SWAYNE, S. L.; PARENT, J. M. Recurrence of thoracolumbar intervertebral disk extrusion in chondrodystrophic dogs after surgical decompression with or without prophylactic fenestration: 265 cases (1995-1999). Journal of the american veterinary medicine association, New York, v.224, p.1808-1814, 2004.

7. CHIERICHETTI A.L.; ALVARENGA, J. Afecção degenerativa do disco intervertebral toracolombar: revisão. Clínica Veterinária. v.22, p. 25-30, 1999.

8. CHRISMAN, Cheryl L. Neurologia dos pequenos animais. São Paulo: Roca, p.354-360, c1985 432p.

9. DE LAHUNTA, A. Veterinary neuroanatomy and clinical neurology. 2 . ed. Philadelphia: Saunders, 1983, 471 p.

10. ERNST, E.. Chiropractic: A Critical Evaluation. Journal of Pain and Symptom Management: 2008 preprint. Página visitada em 2011-05-25.

11. GARIBALDI, L. Síndrome da cauda eqüina In PELEgRINo, F.; SURANITI, A. Síndromes Neurológicas em cães e gatos. São Caetano do Sul: Interbook, p. 110$115,2003$.

12. HELLYER, P.W.; ROBERTSON, S.A.; FAILS, A.D. Pain and its management. In: TRANQUILLI, W.J.; THURMON, J.C.; GRIMM, K.A. (Eds). Lumb \& Jones's Veterinary Anesthesia and Analgesia. 4ed. Iowa: BlackwellPublishing Professional, 2007. p.31-57.

13. HYUNJUNG, H.; SOONWUK, J.; JOONYOUNG, K.; MANBOK, J.; JESUN, K. The effect of conservative therapy on thoracolumbar intervertebral disc disease on 15 dogs. $\mathrm{J}$. Vet. Clin., v.20, p.52-58, 2003.

14. JANSSENS, L.A.A. Acupuncture for thoracolumbar and cervical disk disease. In: SCHOEN, A. M. Veterinary Acupuncture - Ancient Art to Modern Medicine. 2 ed. St. Louis: Mosby, 2001. cap. 14, p. 193-198.

15. JEFFERY, N. D. Handbook of small animal spinal Surgery. London. Saunders, p. 202229. 1995

16. JOAQUIM, Jean G. F. Comparação entre eletroacupuntura, cirurgia e cirurgia associada à eletroacupuntura no tratamento da doença do disco intervertebral em cães. Universidade Estadual Paulista. 2008. Tese (Doutorado). Faculdade de Medicina Veterinária e Zootecnia, Botucatu, São Paulo.

17. KONNO, S.; KAYAMA, S.; OLMARKER, K.; KIKUCHI, S. Effects of OP-11206 (prostaglandin E1) on nerve-conduction velocity in the dog cauda equina subjected to acute Bol. Med. vet. - Espírito Santo do Pinhal, v.2, n.2, p.26-33, jan./dez. 2006. 33 experimental compression. Journal of Spinal Disorders, Philadelphia, v.9, p.103$106,1996$.

18. LECOUTEUR, R.A.; CHILD, G. Moléstias da medula espinhal. In: ETTINGER,S.J. Tratado de medicina veterinária: Moléstias do cão e do gato. 3 ed. São Paulo: Manole, 1992. cap.62, p.655-736.

19. MALMEGRIN, P. V. Redação - Acupuntura Veterinária. Disponível em:

<http://www.gaybrasil.com.br/acupunturaveterinaria.asp?Categoria=Idigital\&Codigo=2056 >. Acesso em 09 Setembro 2011.

20. MCKEE, M. W; MITTEN, W. R.; LABUC, H. R. Surgical treatment of lombossacral discospondylitis by a distraction-fusion technique. Journal of Small Animal Practice, Australia, v.31, p.15-20, 1990. 
21. MORIKAWA, Vivien Midori. Síndrome da cauda eqüina em cães. Disponível em: <http://www.sosanimal.com.br/clinica/informativo/exibInformativo/?codigo=93. $>$.

Acesso em: 22 out. 2011.

22. NELSON, Richard W.; COUTO, C. Guillermo. Medicina interna de pequenos animais. 3.ed. Rio de Janeiro: Elsevier, p.1002-1004, 2006. 1324p.

23. PALMER, R. H.; CHAMBERS, J. N. Canine lombossacral diseases. PartI, Anatomy, pathophysiogy, and clinical presentation. Compendium on Continuing Education for the Practicing Veterinarian, v.19, p.61-69, 1991.

24. PINTO, Ana Carolina B. de C. F. et al. Aspectos radiográficos e tomográficos de hemangiossarcoma de meninges causando síndrome da cauda eqüina em um Pastor Alemão. Ciência Rural, Santa Maria, v.37, n.2, mar./abr. 2007.

25. PRATA, R. G. Afecções da Coluna Lombossacral: In SLATTER, D. Manual de cirurgia de pequenos animais. 2.ed. São Paulo: Manole, p.1314-1331. 1998.

26. RAMIREZ, O.; THRALL, D. E.A review of imaging techniques for canine cauda equina syndrome. Veterinary Radiology \& Ultrasound, v.39, n.4, p.283-296, Jul. 1998.

27. RENALVET. Quiroprática em Medicina Veterinária. (on line).Disponível em: $<$ http://www.renalvet.com.br/index1.php?topic=artigo1\&idioma=portugues\&cd_seleciona do=22>. Acesso em 10 de junho2011.

28. SCHOEN, A. M. Veterinary acupuncture, ancient art to modern medicine. St. Louis: Mosby, 1994. 707p.

29. SCHULMAN, A. J.; LIPPINCOTT, C. L. Cauda equina syndrome in dogs. Compendium on continuing Education for the practicing Veterinarian, v.10, p. 835-844, 1990.

30. SEIM III H. B.; Fundamentos da Neurocirurgia, In FOSSUM et al; Cirurgia de Pequenos Animais; São Paulo; Primeira Edição; Roca; 1997.

31. SEIM III, H.B. Cirurgia da espinha toracolombar. In: FOSSUM, T.W.; HEDLUND, C.S.; HULSE, D.A.; JOHNSON, A.L.; SEIM III, H.B.; WILLARD, M.D., CARROLL, G.L. Cirurgia de Pequenos Animais. São Paulo: Roca, 2002. cap. 35, p. 1216-1248.

32. SHORES, A.; TEPPER, L. C. A modified ventral approach to the atlantoaxial junction in the dog. Veterinary surgery, Philadelphia, v.36, p.765-770, 2007.

33. SIMPSON, S.T. Intervertebral disc disease. Vet. Clin. North Am. Small Anim. Pract., v.22, p.889-998, 1992.

34. STILL, J. Acupuncture treatment of thoracolumbar disc disease: a study of 35 cases. Comp. Anim. Pract. Acup., v.2, p. 19-24, 1988a.

35. STILL, J. Acupuncture treatment of grade III and IV canine thoracolumbar disc disease (hind limb paralysis). Am. J. Acup., v. 26, p. 179-187, 1998b.

36. STILL, J. Analgesic effects of acupuncture in thoracolumbar disc disease in dogs. J. Small Anim. Pract., v. 30, p.298-301, 1989.FREIRE, M. N. Recursos Fisioterapêuticos Utilizados em Pequenos Animais. 2005. Disponível em: http://www.vetphysical.com.br/artigos. Acesso em 10 Setembro 2011.

37. SLOCUM, B.; DEVINE, T. L7-S1 fixation-fusion for treatment of cauda equine compression in the dog. Journal of the American Veterinary Medical Association, v. 188, p.31-35, 1986.

38. TAGA, Atsuo et al. The advantage of magnetic resonance imaging in diagnosis of cauda equina syndrome in dogs. Journal of Veterinary Medical Science, Japão, v. 60, n. 2, dez. 1998.

39. TAYLOR, S. M. Distúrbios da Medula Espinhal. In NELSON R. W.; COUTO, C. G. Medicina Interna de Pequenos Animais. 3. ed. Rio de Janeiro: Elsevier, 2006. p 994 a 1002. 
40. TOOMBS, J.P. Cervical intervertebral disk disease in dogs. Continuing Education for the practicing Veterinarian, v.14, n.11, p. 1477-1489, 1992.

41. TROTTER, G.W., EMBERTSON, RM. Surgical diseases of the cranial reproductive tract. In: Equine surgery. Philadelphia: WB Saunders, 1992. p.752-754.

42. WHEELER, S.J; SHARP, N. J. H. Diagnóstico e tratamento cirúrgico das afecções espinais do cão e gato. São Paulo: Manole, p.122-134. 1999.

43. WONG, A.M.; LEONG, C.P.; SU, T.Y.; YU, S.W.; TSAI, W.C.; CHEN, C.P. Clinical trial of acupuncture for patients with spinal cord injuries. Am. J. Phys. Med. Rehabil., v.82, p.21-7, 2003. 